\title{
Coronavirus e ambiente: una sfida per il pianeta e per la salute
}

\author{
Cristiano Magnaghi ${ }^{1}$, Elena Brioni ${ }^{2}$, Donato Leopaldi ${ }^{3}$, Marisa Pegoraro ${ }^{4}$, Giuseppe Vezzoli ${ }^{5}$, Paolo Manunta ${ }^{2}$, \\ Maria Teresa Parisotto ${ }^{6}$
}

${ }^{1}$ U.O. Nefrologia e Dialisi, IRCCS Ospedale San Raffaele, Milano - Italia

Università Vita-Salute San Raffaele, Milano - Italia

${ }^{3}$ U.O. Nefrologia e Dialisi, ASST Fatebenefratelli Sacco, Milano - Italia

${ }^{4}$ U.O. Nefrologia e Dialisi, ASST Grande Ospedale Metropolitano Niguarda, Milano - Italia

IRCCS Ospedale San Raffaele, Milano - Italia

${ }^{6}$ Board Member of European Specialist Nurses Organization (ESNO), Bruxelles - Belgio

\begin{abstract}
Coronavirus and environment: a challenge for the planet and the health
In late December 2019, some patients were hospitalized with an initial diagnosis of pneumonia of unknown etiology. These patients were epidemiologically linked to a wholesale market of seafood, wild animals and meat located in Wuhan, Hubei province, China. Acute respiratory disease caused by a new Coronavirus SARS-CoV-2, formerly known as 2019-nCoV, afterwards as Coronavirus disease 2019 (COVID-19), spread throughout China and received the attention of the entire world. Analysis of the genomic sequence of COVID-19 showed $88 \%$ of identity with two bat-derived severe acute respiratory syndrome-like Coronaviruses. Illegal and sustainable wildlife trade is the second largest direct threat to species after habitat destruction. Each year, hundreds of millions of plants and animals are captured or collected in the wild to be sold as food, pets, medicines, ornaments and for a variety of other reasons. Even the climate crisis could offer dangerous scenarios in addition to another fundamental chapter of the environmental pillar which is the one concerning air quality. There is a solid scientific literature which correlates the incidence of viral infection cases with concentrations of atmospheric particulate matter (e.g. PM10 and PM2.5). Despite the current regulations, which limit the levels of certain atmospheric pollutants, there are still numerous negative health effects deriving from exposure to these agents. However, it can be said that chronic air pollution, such as peaks of concentration of fine dust and other pollutants, acts as a pejorative factor in cases of epidemics.
\end{abstract}

Keywords: Air Pollution, Coronavirus, Environment, Spillover, Viral Infection

Alla fine di dicembre 2019, in Cina, un gruppo di pazienti è stato ricoverato in ospedale con una diagnosi iniziale di polmonite di eziologia sconosciuta. Questi pazienti erano epidemiologicamente legati a un mercato all'ingrosso di frutti di mare, animali selvatici e carne a Wuhan, nella provincia di Hubei, in Cina $(1,2)$. La malattia respiratoria acuta, causata da questo nuovo Coronavirus (SARS-CoV-2, precedentemente

Received: April 19, 2020

Accepted: April 20, 2020

Published online: May 13, 2020

Indirizzo per la corrispondenza:

Elena Brioni

IRCCS Ospedale San Raffaele

Via Olgettina 60

20132 Milano - Italia

brioni.elena@hsr.it noto come 2019-nCoV e, poi, come malattia Coronavirus 2019, COVID-19), si è diffusa in seguito in tutta la Cina e ha ricevuto attenzione in tutto il mondo. II 30 gennaio 2020, I'Organizzazione Mondiale della Sanità (OMS) ha dichiarato ufficialmente l'epidemia COVID-19 come un'emergenza di sanità pubblica di interesse internazionale (3).

I sintomi dell'infezione da COVID-19 compaiono dopo un periodo di incubazione di circa 5 giorni (4). Il periodo dall'esordio dei sintomi della malattia COVID-19 alla morte varia da 6 a 41 giorni, con una mediana di 14 giorni. I sintomi più comuni all'inizio della malattia COVID-19 sono febbre, tosse e affaticamento, mentre altri sintomi includono produzione di espettorato, mal di testa, emottisi, diarrea, dispnea e linfopenia (5).

Sulla base dei risultati del sequenziamento del genoma del virus e dell'analisi evolutiva all'origine del nuovo Coronavirus c'è il fenomeno dello spillover, il "salto", che permette al patogeno di passare da una specie all'altra. In questo caso, 
I'analisi della sequenza genomica di COVID-19 ha mostrato I'88\% di identità con due Coronavirus simili alla sindrome respiratoria acuta grave derivati dai pipistrelli, indicando che questi mammiferi sono il legame più probabile tra COVID-19 e I'uomo (6). Una trasmissione efficiente da uomo a uomo è un requisito fondamentale per la diffusione su larga scala di un nuovo virus. II contagio da persona a persona avviene principalmente attraverso il contatto diretto o attraverso le goccioline che si diffondono se un individuo infetto tossisce o starnutisce (7).

Sappiamo che il $60 \%$ di tutte le malattie infettive nell'uomo è di origine zoonotica (8) e la Cina ha una lunga storia di mercati di animali vivi considerati vitali per le comunità di tutto il paese. Pertanto, è improbabile che questi mercati verranno chiusi definitivamente, sebbene la loro chiusura sarebbe il deterrente più forte per un'altra epidemia di malattia zoonotica. La sorveglianza di questi mercati può essere vitale per controllare la diffusione delle malattie zoonotiche e dovrebbero essere adottate misure appropriate per garantire che vengano utilizzati protocolli sanitari e igienici che limitino il contatto tra animali vivi ed esseri umani (9).

Il commercio illegale e sostenibile della fauna selvatica è la seconda più grande minaccia diretta alle specie dopo la distruzione dell'habitat. La distruzione dell'habitat per mano umana rompe gli equilibri biologici e annulla ogni tipo di barriera naturale, le modifiche apportate dall'uomo per nuove edificazioni, la creazione di nuovi pascoli e la produzione di carta e legname e cancellano parte di quelle specie animali che rappresentano un argine tra i virus e l'essere umano, aumentando, così, i rischi di contagio. Colpisce, in particolare, i più poveri del mondo che dipendono da animali selvatici locali per la carne e da alberi e piante locali per cibo e medicine.

Se, per il Coronavirus, il meccanismo identificato è quello di un "salto" di specie innescato dalla promiscuità con animali selvatici unito alla concentrazione di popolazione nelle megalopoli e al trasporto dato dalla globalizzazione, la crisi climatica potrebbe, invece, offrire scenari ancora più pericolosi. Per tale motivo, un altro capitolo fondamentale del pilastro "ambiente" è quello che concerne la qualità dell'aria.

Riguardo agli studi sulla diffusione dei virus nella popolazione vi è una solida letteratura scientifica che correla l'incidenza dei casi di infezione virale con le concentrazioni di particolato atmosferico (p. es., PM10 e PM2,5). È noto che il particolato atmosferico funziona da carrier, ovvero da vettore di trasporto, per molti contaminanti chimici e biologici, inclusi i virus (10).

Nonostante le normative vigenti, che limitano i livelli di determinati inquinanti atmosferici, sussistono ancora numerosi effetti negativi sulla salute derivanti dall'esposizione a questi agenti.

Pochi studi si sono concentrati sulla possibile associazione tra inquinamento e malattie trasmissibili. Inoltre, in concomitanza con il rapido sviluppo economico e con l'urbanizzazione, l'inquinamento atmosferico è diventato un grave problema e rappresenta una grave minaccia per la salute della popolazione.

Cui et al. (11), in uno studio ecologico condotto tra il $2002 \mathrm{e}$ il 2003 rispetto alla sindrome respiratoria acuta grave (SARS), che ha causato 349 morti con 5327 casi probabili segnalati nella Cina continentale, dimostravano un'associazione positiva tra livelli di indice di inquinamento atmosferico (API) e mortalità. L'analisi ecologica condotta tra 5 regioni con 100 o più casi di SARS ha mostrato che il tasso di mortalità del caso è aumentato con l'incremento dell'indice di inquinamento atmosferico (mortalità del caso $=-0,063+0,001 *$ API). Uno studio parzialmente ecologico basato sull'esposizione a breve termine ha dimostrato che i pazienti con SARS provenienti da regioni con API moderati avevano un rischio aumentato dell' $84 \%$ di morire di SARS rispetto a quelli provenienti da regioni con API bassi ( $R R=1,84$, IC al 95\%: 1,41-2,40).

Uno studio multicentrico del 2017 (12), condotto in 47 città della Cina, analizzava, comparando i dati sull'influenza, le misurazioni al suolo di PM2,5 e i dati meteorologici, l'impatto delle particelle fini ambientali sulla trasmissione dell'influenza e gli effetti di modifica della temperatura. Lo studio dimostrava che, complessivamente, il 10,7\% dei casi di influenza può derivare dall'esposizione al PM2,5 ambientale e che la trasmissione influenzale associata al PM2,5 era modificata in base alle condizioni meteorologiche. L'esposizione al PM2,5 è associata a una vasta gamma di malattie, tra cui quelle cardiovascolari e quelle respiratorie (13).

Le azioni dell'uomo e alcune malattie hanno un impatto sulla salute delle persone e sull'aspetto economico e sociale. Attraverso piani di sviluppo responsabili e sostenibili, con particolare occhio di riguardo ai cambiamenti climatici e a sistemi che garantiscano uno sviluppo quanto più naturale possibile, il genere umano potrà proliferare in armonia con l'ambiente che lo circonda. Per quanto non si possa arrivare a una conclusione generale quantitativa, dato che i diversi studi valutano contesti specifici sui quali giocano molti fattori diversi, si può, però, affermare che l'inquinamento cronico dell'aria e i picchi di concentrazione di polveri sottili e di altri inquinanti agiscano come fattori peggiorativi in caso di epidemia. Ed è perfettamente plausibile che ciò avvenga, sia sotto forma di possibile veicolo che amplifica la diffusione del virus sia come fattore di stress cronico che potrebbe rendere più vulnerabile la popolazione agli effetti dell'epidemia; attualmente, però, non è ancora possibile, nel caso italiano, stabilire di quanto.

\section{Disclosures}

Conflict of interest: The authors have no conflict of interest. Financial support: The authors declare no financial support.

\section{Bibliografia}

1. Bogoch A, Watts A, Thomas-Bachli A, et al. Pneumonia of unknown etiology in Wuhan, China: potential for international spread via commercial air travel. J Travel Med. 2020;27(2). 
2. Lu H, Stratton CW, Tang YW. Outbreak of pneumonia of unknown etiology in Wuhan China: the mystery and the miracle. J Med Virol. 2020;92(4):401-2.

3. Guo $Y, C a o Q$, Hong $Z$, et al. The origin, transmission and clinical therapies on coronavirus disease 2019 (COVID-19) outbreak an update on the status. Mil Med Res. 2020;7(1):11. https:// doi.org/10.1186/s40779-020-00240-0.

4. Li Q, Guan X, Wu P, et al. Early transmission dynamics in Wuhan, China, of novel coronavirus-infected pneumonia. N Engl J Med. 2020;382:1199-207.

5. Huang $\mathrm{CY}$, Wang $\mathrm{Y}$, Li $\mathrm{X}$, et al. Clinical features of patients infected with 2019 novel coronavirus in Wuhan, China. Lancet. 2020;395:497-506.

6. Wan Y, Shang J, Graham R, et al. Receptor recognition by novel coronavirus from Wuhan: an analysis based on decade-long structural studies of SARS. J Virol. 2020;94(7):e00127-20.

7. Munster VJ, Koopmans M, Van Doremalen N, et al. A Novel Coronavirus Emerging in China - Key Questions for Impact Assessment N Engl J Med. 2020;382(8):692-4.

8. Woolhouse ME, Gowtage-Sequeria S. Host range and emerging and reemerging pathogens. Emerg Infect Dis. 2005;(11):1842-7.
http://www.ncbi.nlm.nih.gov/pmc/articles/PMC3367654/pdf/ 05-0997.pdf.

9. Peeri NC, Shrestha N, Siddikur Rahman MD, et al. The SARS, MERS and novel coronavirus (COVID-19) epidemics, the newest and biggest global health threats: what lessons have we learned? Int J Epidemiol. 2020;dyaa033. https://doi.org/ 10.1093/ije/dyaa033.

10. Ciencewicki J, Jaspers I. Air pollution and respiratory viral infection. Inhal Toxicol. 2007;19(14):1135-46.

11. Cui Y, Zhang Z, Froines J, et al. Air pollution and case fatality of SARS in the People's Republic of China: an ecologic study. Environ Health. 2003;2(1):15. https://doi.org/10.1186/1476069X-2-15.

12. Chen $\mathrm{G}$, Zhang $\mathrm{W}$, Li S, et al. The impact of ambient fine particles on influenza transmission and the modification effects of temperature in China: A multi-city study. Environ Int. 2017;(98):82-8.

13. Arnold C. Disease burdens associated with PM2.5 exposure: how a new model provided global estimates. Environ Health Perspect. 2014;122(4):A111. 